\title{
On a 2D Model of Avascular Tumor with Weak Allee Effect
}

\author{
Peng Feng $\left(\mathbb{D},{ }^{1}\right.$ Zhewei Dai, $^{2}$ and Dorothy Wallace $\mathbb{D I}^{3}$ \\ ${ }^{1}$ Department of Mathematics, Florida Gulf Coast University, 11501 FGCU Blvd. S., Fort Myers, FL 33965, USA \\ ${ }^{2}$ Department of Mathematics, Alma College, 614 W Superior St, Alma, MI 48801, USA \\ ${ }^{3}$ Department of Mathematics, Dartmouth College, Hanover, NH 03755, USA \\ Correspondence should be addressed to Peng Feng; pfeng@fgcu.edu
}

Received 17 December 2018; Revised 25 April 2019; Accepted 22 May 2019; Published 1 July 2019

Academic Editor: Elisa Francomano

Copyright (C) 2019 Peng Feng et al. This is an open access article distributed under the Creative Commons Attribution License, which permits unrestricted use, distribution, and reproduction in any medium, provided the original work is properly cited.

\begin{abstract}
Recent studies reveal that Allee effect may play important roles in the growth of tumor. We present one of the first mathematical models of avascular tumor that incorporates the weak Allee effect. The model considers the densities of tumor cells in three stages: proliferating cells, quiescent cells, and necrotic cells. We investigate how Allee effect impacts the growth of the avascular tumor. We also investigate the effect of apoptosis of proliferating cells and necrosis of quiescent cells. The system is numerically solved in $2 \mathrm{D}$ using different sets of parameters. We show that Allee effect and apoptosis play important roles in the growth of tumor and the formation of necrotic core.
\end{abstract}

\section{Introduction}

Genetic mutation in DNA modifies the normal cell proliferation rate and causes a normal cell to become cancerous. A cancer cell then searches for nutrient such as oxygen and glucose from nearby tissues and proliferates rapidly. This results in a clump of cancer cells known as tumor. The growth of a tumor depends on many factors such as its location, cell type, and also the availability of nutrients. There are three stages of tumor growth, namely, avascular stage, angiogenesis stage, and metastasis stage ([1]). At avascular stage, the tumor often grows in an almost spherical form (Figure 1). At this stage, the tumor is harmless and may reach a saturation size due to the limited nutrient supply to the core of the tumor. However, nutrient-deficient tumor cells produce signaling proteins such as vascular endothelial growth factor (VEGF). When enough such signaling proteins are produced, the nearby blood vessels will provide additional nutrient through neovasculature to sustain the growth of the tumor, leading to the second stage called angiogenesis. Once the tumor makes connection with nearby blood vessels, mobile tumor cells can migrate to the other parts of the body and develop additional tumors at different sites, resulting in the metastasis stage ([2]).

In experiments, tumor cell lines may be grown in many different ways, for example, as a two dimensional monolayer on a substrate, as a three dimensional spheroid suspended in a liquid or gel, and as a mouse xenograft. Each of these methods yields different information about the cell line. For example, it is known that the response of the tumor culture to treatment differs between monolayer and spheroid cultures ([3]), and mathematical models exhibit this differential response also ([4]). Glioma cells grown as spheroids produce more lactic acid, use more glucose, and exhibit more markers of hypoxia than those grown in monolayer.

There are multiple methods for growing spheroids ([5]). Tumor cell lines that are adhesive enough to be grown as in vitro spheroids always exhibit a necrotic core ([6]). It is commonly believed that the necrotic core is formed when the oxygen drops to a critical level, although it is also known that a feedback mechanism due to the production of tumor necrosis factor alpha causes apoptosis in the proliferating cell population $([7,8])$. Both mechanisms are supported by the observation that when the tumor spheroids grown in vitro have a diameter reaching $500 \mu \mathrm{m}$, a necrotic core will form ([9]), and their growth eventually ceases ([10]). Both mechanisms are also supported by assorted simple mathematical models ([11]). In support of factors besides hypoxia, some researchers observed that necrotic core can also form in relatively high oxygen level $([12,13])$. In ([13]), MullerKlieser pointed out that necrotic core is not necessarily a consequence of hypoxia or anoxia but emerges as an accumulation of apoptosis. Apoptotic fragments may accumulate 


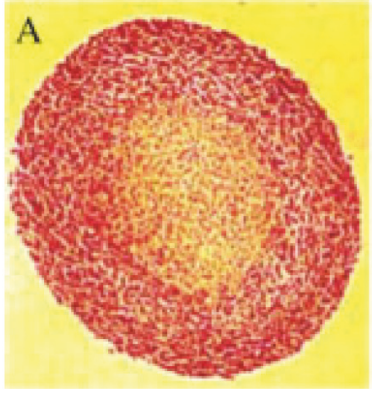

(a)

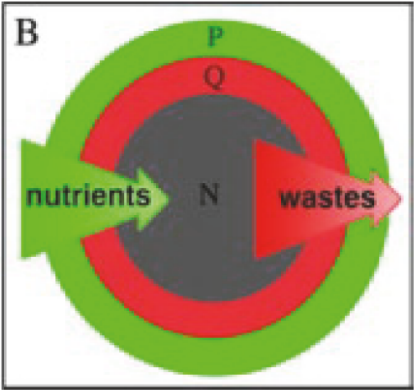

(b)

FIGURE 1: Structure of a typical tumor spheroid: a proliferating layer in the outer rim and an inner core of necrotic cells, separated by a layer of quiescent cells.

in the tumor, leading to the formation of a necrotic core. They observe that the diameter of the necrotic core expands at approximately the same rate as the spheroid volume increases. This results in a relatively constant thickness of the viable cell rim with increasing spheroid diameter ([12]). In this article, we consider both hypoxia-induced apoptosis and necrosis as mechanisms that lead to the formation of central necrotic core, but leave the role of tumor necrosis factor alpha for a future study, although it is known that glioma cells are sensitive to TNF-alpha ([14]). See Figure 2 for a comparison between apoptosis and necrosis.

In recent years, there has been a growing interest in viewing tumors and its microenvironment as an ecosystem $([15,16])$. A cancer ecosystem contains multiple strains of tumor cells as well as epithelial cells, nutrients, growth factors, etc. This perspective emphasizes their interactions and consequences ([17]) instead of treating cancer as a collection of muted cells. Our knowledge of ecological dynamics within tumors is limited, especially in small tumors prior to detection or after treatment ([16]). However, even under the assumption that a single cell line is grown in a biologically inert substrate, ecological phenomena may occur, in particular, the Allee effect. First described in the 1930s, Warder Allee was able to show that, contrary to intuition, goldfish grow more rapidly in a tank with more goldfish ([18]). Since then, ecologists and mathematicians have studied this effect, now termed Allee effect, in many different situations. A strong Allee effect describes a population that can grow at intermediate population density but declines when the number of organisms is either too small or too large. Such populations are then likely to collapse and become extinct if their population size falls below a certain threshold. Clearly, any cancer cell lines that can propagate indefinitely in vitro are highly unlikely to be subject to a strong Allee effect. However, a weak Allee effect describes a population that grows with a reduced per capita growth rate at a low population size, although the growth rate is still positive. For example, lowgrade cell line cultures, in vivo and in vitro, have low chances of persistence and low reproducibility. On the contrary, highgrade cell lines have a higher chance to establish $([19,20])$. The possible existence of a weak Allee effect in tumor cells has been described in a very recent study ([21]). The proliferation rate of tumor cells at low density was measured via a series of in vitro experiments with two glioblastoma cell lines ([22]), where it was found that when the cell density is low, the growth rate increases with the population density, while decreasing at larger density. This study, which suggests a weak Allee effect in glioblastoma, is echoed in some cellular automata models of spheroid growth, in which low density affects slow proliferation ([23]). In cultures of cancer cells weak Allee effect likely arises due to autocrine growth factors, diffusive signaling molecules produced and secreted by cells that enhance growth and proliferation of other cells ([22]).

The study of tumor growth using mathematical models has been in focus for the past four decades or so. Starting from early diffusion and differential equation models by Thomlinson and Gray, which was then extended by Burton and Greenspan, tumor models have expanded to include ordinary differential equation (ODE) models, partial differential equation (PDE) models, cellular automata (CA) models, and the more sophisticated hybrid models that combines PDE models and CA models ([24-26]). For a complete review on mathematical modeling of avascular tumors, we refer the readers to the review by Araujo and McElwain ([27]) or the more recent one by ([28]). Here we describe briefly a handful of models closely related to the one presented in this study.

A malignant tumor invasion model with strong Allee effect was recently studied in ([29]), focusing on properties of travelling wave solutions. A PDE system comprising both live-cell density, dead-cell density, and nutrient density was developed in ([30]). However, they did not distinguish between proliferating and quiescent cells, nor did they consider different types of cell death (via necrosis or apoptosis). Later they built upon this model by incorporating two necrotic depletion mechanisms: leakage of the dead cellular material by diffusion or consumption of cellular material for the construction of new cells ([31]).

Sherratt and Chaplain proposed an interesting model that accounts for three different states of tumor cells ([32]). In their model, the necrotic core comes directly from the necrosis of quiescent cells and there is no weak Allee effect. Their model also does not consider the fact that quiescent cells 


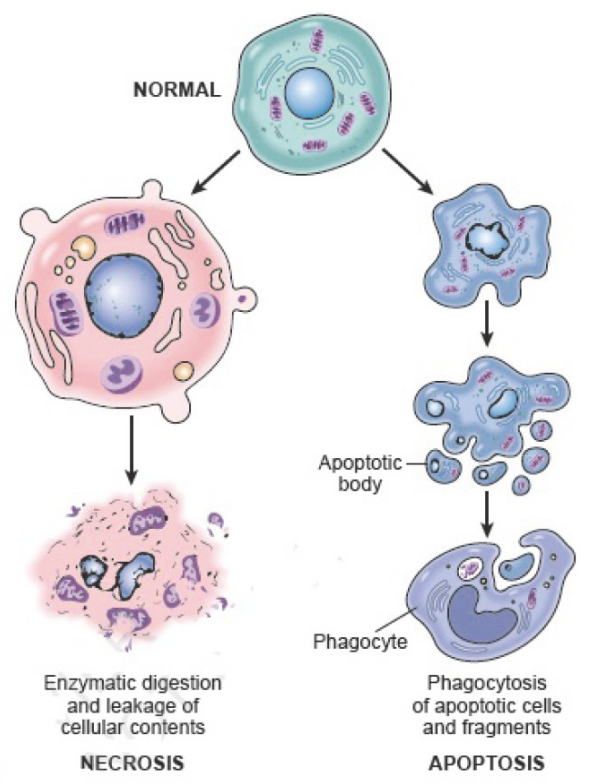

Figure 2: Difference between necrosis and apoptosis.

consume nutrients as well, only at a slower rate. Including cell movement due to contact inhibition, they considered the following reaction diffusion model in $1 \mathrm{D}$ :

$$
\begin{aligned}
\frac{\partial P}{\partial t}= & \frac{\partial}{\partial x}\left[\frac{P}{P+Q} \frac{\partial}{\partial x}(P+Q)\right] \\
& +g(c) P(1-P-Q-N)-f(c) P, \\
\frac{\partial Q}{\partial t}= & \frac{\partial}{\partial x}\left[\frac{Q}{P+Q} \frac{\partial}{\partial x}(P+Q)\right]+f(c) P-h(c) Q \\
\frac{\partial N}{\partial t}= & h(c) Q \\
\frac{\partial C}{\partial t}= & D_{c} \Delta C+k_{1} C_{0}[1-\alpha(P+Q+N)]-k_{1} C \\
& -k_{2} C P .
\end{aligned}
$$

The existence of travelling wave solutions for some special cases was studied in Zhu and $\mathrm{Ou}$ [33] using the Banach fixed point theorem and perturbation methods. Travelling wave solutions to similar systems for contact inhibition were also studied in Bertsch et al. [34]. The impact of Allee effect on the spreading of species has been well studied in ecological modeling. It is generally believed that Allee effect can adversely affect the spreading speed. The Allee effect can also explain the time lag that is often noticed between the time when a species first establishes and the time it starts to spread rapidly ([35]). Similarly, an Allee effect caused a long time lag between the resection and recurrence in a one dimensional spatial model glioblastoma ([22]).

The early study of the travelling wave solution of the following reaction diffusion equation with Allee effect

$$
u_{t}=u_{x x}+u(1-u)(u-\beta)
$$

can be found in $([36,37])$. beta represents the Allee threshold below which the growth rate of the species becomes negative. The main results can be summarized as follows: (a) For $0<$ $\beta<1$, there exists a unique wave front solution with speed $c=$ $\sqrt{2}(\beta-1 / 2)$. For $\beta<1 / 2$, the front propagates to the region where the species is absent, i.e., the invasion of the species; for $\beta>1 / 2$, the front propagates to the region where the species is at its carrying capacity, i.e., the retreat of the species; when $\beta=1 / 2$, the front is stationary.

(b) For $-1<\beta \leq 0$, there exists a maximal wave speed such that the travelling wave solution exists when $c \leq c^{*}$. Moreover, $c^{*}=-2 \sqrt{-\beta}$ for $-1<\beta \leq-1 / 2$ (derived by linearizing about zero) and $c^{*}=\sqrt{2}(\beta-1 / 2)$ when $-1 / 2<$ $\beta \leq 0$.

A weak Allee effect in a highly necrotic expanding spheroid would necessarily apply to any spatial region where the density of live cells is quite low. This is a situation where mathematical modeling can reveal unknown or counterintuitive principles that may have been overlooked before. It can test theories by simplifying the underlying mechanisms ([38]). Our model builds on prior ones by extending the spatial domain to two dimensions, including diffusion of nutrient, motion of cells, the weak Allee effect, the quiescent cell compartment, and two mechanisms of cell death: apoptosis and necrosis. Our hypothesis is that, for expanding spheroids in substrate suitable for migration, a weak Allee effect will slow the rate of expansion of the proliferating edge, as measured by the speed of the wavefront. To capture the spatial characteristics of central necrosis, a ring of quiescent cells, and a proliferating rim developing in response to nutrient delivery and depletion we propose a system of PDEs in two spatial dimensions, and with weak Allee effect, for the purpose of computationally testing this hypothesis. 


\section{Mathematical Model}

Consider the following model:

$$
\begin{aligned}
\frac{\partial P}{\partial t}= & \nabla \cdot\left(\frac{P}{P+Q} \nabla(P+Q)\right) \\
& +g(C) P(P+m)(1-P-Q-N) \\
& -f_{1}(C) P+f_{2}(C) Q-f_{3}(C) P, \\
\frac{\partial Q}{\partial t}= & \nabla \cdot\left(\frac{Q}{P+Q} \nabla(P+Q)\right)+f_{1}(C) P-f_{2}(C) Q \\
& -h(C) Q \\
\frac{\partial N}{\partial t}= & h(C) Q+f_{3}(C) P, \\
\frac{\partial C}{\partial t}= & D_{c} \Delta C+k_{1} C_{0}[1-\alpha(P+Q+N)]-k_{1} C \\
& -k_{2} C P-k_{3} C Q,
\end{aligned}
$$

subject to the initial conditions

$$
\begin{aligned}
& P(0, x, y)=P_{0}(x, y), \\
& Q(0, x, y)=0, \\
& N(0, x, y)=0, \\
& C(0, x, y)=C_{0},
\end{aligned}
$$

and boundary conditions

$$
\begin{aligned}
& (P, Q, N, C) \longrightarrow\left(0,0,0, C_{0}\right) \\
& \text { when } r=\sqrt{x^{2}+y^{2}} \longrightarrow \infty,
\end{aligned}
$$

$(\nabla P, \nabla Q, \nabla N, \nabla C) \longrightarrow(0,0,0,0)$

$$
\text { when } r=\sqrt{x^{2}+y^{2}} \longrightarrow \infty \text {. }
$$

Here $P$ represents the density of proliferating cells, $Q$ the density of quiescent cells, and $N$ the density of necrotic cells. $C$ denotes the concentration of oxygen.

The functions $f_{1}(C)$ and $h(C)$ will be decreasing functions of $C$ with $f_{1}(+\infty)=h(+\infty)=0 . g(c)$ is an increasing function of $C$. $f_{2}(C)$ will be increasing with $f_{2}(0)=0$. $f_{3}(C)$ is the apoptotic rate at which proliferating cells die. For simplicity, we assume $f_{3}(C)$ is a constant. The exact forms of these switching functions remain unknown. Many authors have considered the simplest case, for example, a linear switching rate between proliferating cells and quiescent cells. $f_{1}(C)$ is the rate at which proliferating cells become quiescent. We use a Michaelis-Menten-type expression

$$
f_{1}(C)=K_{P}\left(1-\frac{C^{m_{1}}}{C_{c r i t 1}^{m_{1}}+C^{m_{1}}}\right),
$$

where $C_{\text {crit } 1}$ is the critical oxygen level and $m_{1}$ is positive constant which governs the sharpness of change $f_{1}(C)$ near

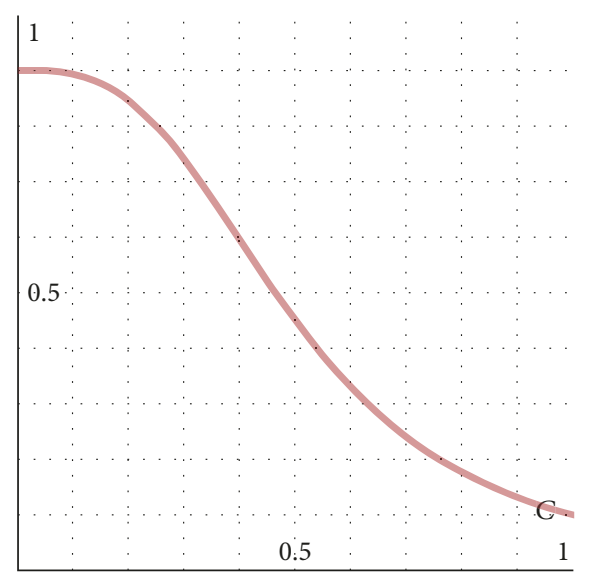

FIGURE 3: A typical profile of $f_{1}(c)$ with $K_{P}=0.9, m_{1}=3$, and $c_{\text {crit } 1}=0.5$.

the critical concentration. $K_{P}$ is the switching rate when the oxygen is 0 . In the numerical simulations that follow, we take $K_{P}=0.9$ as the standard value. A typical profile of $f_{1}(C)$ is presented in Figure 3.

For the switching rate from quiescent cells back to proliferating cells, we take

$$
f_{2}(C)=\frac{K_{Q} C^{m 1}}{C_{c r i t 1}^{m_{1}}+C^{m_{1}}}
$$

where $K_{Q}$ is switching rate when $c$ is high. In the simulations that follow, we take $K_{Q}=0.05$ as the standard value. See Figure 4 for a typical profile of $f_{2}(C)$.

For the apoptotic rate, we let

$$
f_{3}(C)=K_{N}
$$

where $0 \leq K_{N} \leq 1$ is the apoptosis rate of proliferating cells.

For the necrosis rate of quiescent cells, we adopt the following form

$$
h(C)=K_{D}\left(1-\frac{C^{m_{1}}}{C_{c r i t 2}^{m_{1}}+C^{m_{1}}}\right),
$$

where $0<K_{D}<1$ is the maximum necrosis rate of quiescent cell. $C_{\text {crit } 2}$ is the critical oxygen level for necrosis. See Figure 5.

We let $g(C)=\beta e^{\beta C}$ with $\beta=0.5$.

As $m_{1} \longrightarrow \infty, f_{1}$ tends to a Heaviside function. These two choices of switching function do not significantly alter the outcome as their profiles can become very similar when $m_{1}$ is large.

The oxygen diffuses according to a diffusion coefficient $D_{c}$. The degradation rate of oxygen is denoted by $k_{1}$ and it is utilized by proliferation cells at a rate $k_{2}$. The rate that is being utilized by quiescent cells is denoted by $k_{3}$. Throughout this paper, $k_{3}$ is assumed to be $(1 / 5) k_{2}$, within the experimentally determined range Freyer et al. [39].

Given that the diffusion rate of oxygen throughout the tumor spheroid is much faster comparing to the scale of 


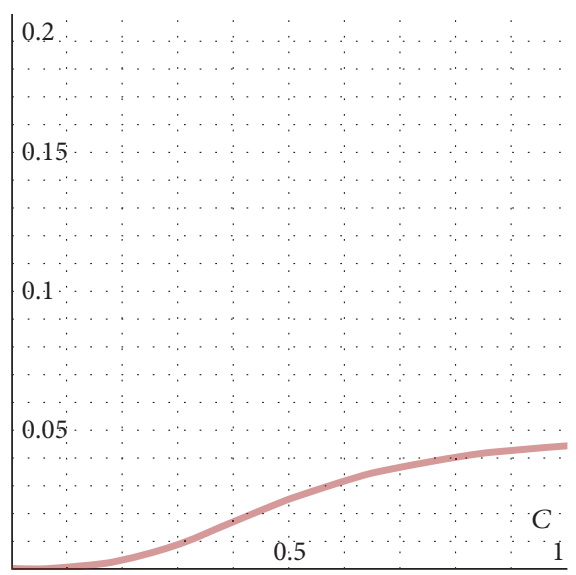

FIgURE 4: A typical profile of $f_{2}(c)$ with $K_{Q}=0.05, m_{1}=3$, and $c_{c r i t 1}=0.5$.

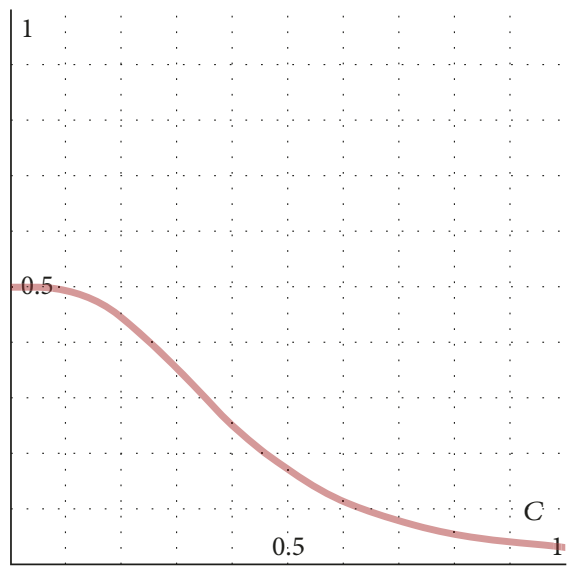

FIGURE 5: A typical profile of $h(c)$ with $K_{D}=0.5, m_{1}=3$, and $c_{c r i t 2}=0.4$.

growth, we adopt the standard quasi steady-state assumption that

$$
C=\frac{C_{0}}{1+\gamma_{1} P+\gamma_{2} Q}(1-\alpha(P+Q+N))
$$

where $\alpha, \gamma_{1}=k_{2} / k_{1}$, and $\gamma_{2}=k_{3} / k_{1}$ are dimensionless parameters. For simplicity, we assume $\gamma_{2}=(1 / 5) \gamma_{1}$.

Our model depends crucially on the biological parameters. These parameters vary greatly depending on tumor type, localization, progression, etc. Since our goal is to investigate the impact of different parameters on the growth of avascular tumors, parameters are chosen within the experimentally observable range rather than a quantitative description of any specific tumor. Most of the parameters involved are available in literature $([40,41])$. Our system is already given in nondimensional form for computational reasons. We take characteristic scale of time and length $T_{0}=10^{4}$ second and $L=0.05 \mathrm{~cm}$, respectively. These nondimensional parameters are summarized in Table 1.

\section{Impact of Allee Effect on Travelling Wave Solution and Wave Speed}

In Petrovskii et al. [42], the authors considered how Allee effect impacts different scenarios of biological invasion in a predator-prey system. They noticed that as a result of the interplay between the Allee effect and the predation, species extinction and unbounded spatial spread are not the only possible system dynamics. For certain parameter values, initial species distribution can evolve to quasi-stationary patches. The position of these patches does not change with time while their shape is either stationary or oscillatory. They pointed out that invasive species can be held localized due to certain interspecific and intraspecific interactions such as the interplay between the Allee effect and predation.

We consider travelling wave solutions of the following form:

$$
\begin{gathered}
P(x, t)=P(z), \\
Q(x, t)=Q(z), \\
N(x, t)=N(z),
\end{gathered}
$$


TABLE 1: Dimensionless parameter values used in our simulation.

\begin{tabular}{lcc}
\hline Parameter & Value & Description \\
\hline$K_{P}$ & 0.9 & Maximum switch rate to quiescent cells \\
$K_{\mathrm{Q}}$ & 0.05 & Maximum switch rate to proliferating cells \\
$K_{D}$ & 0.5 & Maximum necrosis rate \\
$K_{N}$ & 0.01 & Apoptosis rate \\
$C_{c r i t 1}$ & 0.5 & Critical oxygen level for proliferation \\
$C_{c r i t 2}$ & 0.4 & Critical oxygen level for necrosis \\
$k_{1}$ & 0.15 & Oxygen degradation rate \\
$k_{2}$ & 0.3 & Oxygen utilization rate by proliferating cells \\
$k_{3}$ & 0.06 & Oxygen utilization rate by quiescent cells \\
$\gamma_{1}=k_{2} / k_{1}$ & 2 & Positive parameter \\
$\gamma_{2}=k_{3} / k_{1}$ & 0.4 & Positive parameter \\
$\alpha$ & 0.9 & Crowding factor \\
$m$ & 0.4 & Allee effect \\
$m_{1}$ & 3 & Positive parameter
\end{tabular}

where $z=x-a t$ and $a$ is the wave speed. Substituting these solutions into the original system yields the following ODEs

$$
\begin{aligned}
& \left(\frac{P\left(P^{\prime}+Q^{\prime}\right)}{P+Q}\right)^{\prime}+a P^{\prime} \\
& \quad+g(C) P(P+m)(1-P-Q-N)-f_{1}(C) P \\
& \quad+f_{2}(C) Q-f_{3}(C) P=0 \\
& \left(\frac{Q\left(P^{\prime}+Q^{\prime}\right)}{P+Q}\right)^{\prime}+a Q^{\prime}+f_{1}(C) P-f_{2}(C) Q \\
& \quad-h(C) Q=0, \\
& a N^{\prime}+h(C) Q+f_{3}(C) P-d N=0
\end{aligned}
$$

As usual, we linearize the first equation of this system about $P=Q=0$ and assume $P(z)=\bar{P} e^{-\xi z}, Q(z)=\bar{Q} e^{-\xi z}$, and $N(z)=\bar{N} e^{-\xi z}$ to leading orders ([37]); we have

$$
\xi^{2} \bar{P}-a \xi \bar{P}+\left[m g\left(c_{0}\right)-f_{1}\left(c_{0}\right)-f_{3}\left(c_{0}\right)\right] \bar{P}=0 .
$$

Thus for nontrivial solutions, we must have

$$
\xi=\frac{1}{2}\left(a \pm \sqrt{a^{2}-4\left[m g\left(c_{0}\right)-f_{1}\left(c_{0}\right)-f_{3}\left(c_{0}\right)\right]}\right) .
$$

Thus positive solution exists if and only if $a \geq a_{*}$ where

$$
a_{*}:=2 \sqrt{m g\left(c_{0}\right)-f_{1}\left(c_{0}\right)-f_{3}\left(c_{0}\right)} .
$$

Thus the minimal speed $a_{\min }$ (if exists) for our model must satisfy $a_{\min } \geq a_{*}$.

It remains unclear if linear determinacy holds for this problem. By linear determinacy, we mean $a_{\min }=a_{*}$.

\section{Numerical Results and Discussion}

All our numerical simulations employ the zero-flux boundary conditions with a system size $210 \times 210$ discretized through $x \longrightarrow\left(x_{0}, x_{1}, \ldots, x_{n}\right)$ and $y \longrightarrow\left(y_{0}, y_{1}, \ldots, y_{n}\right)$, with $n=$ 210. Our simulations use forward difference in time and central difference in space with a small time step $\tau=0.004$ and space step $h=1$. The concentration at mesh point $\left(x_{i}, y_{j}\right)$ at time $(n+1) \tau$ is denoted by $\left(P_{i, j}^{n+1}, Q_{i, j}^{n+1}, N_{i, j}^{n+1}\right)$ and they are given by

$$
\begin{aligned}
P_{i, j}^{n+1} & =P_{i, j}^{n}+\tau\left(U_{i, j}^{n}+F\left(P_{i, j}^{n}, Q_{i, j}^{n}, N_{i, j}^{n}, C_{i, j}^{n}\right)\right) \\
Q_{i, j}^{n+1} & =Q_{i, j}^{n}+\tau\left(V_{i, j}^{n}+G\left(P_{i, j}^{n}, Q_{i, j}^{n}, N_{i, j}^{n}, C_{i, j}^{n}\right)\right) \\
N_{i, j}^{n+1} & =N_{i, j}^{n}+\tau\left(H\left(P_{i, j}^{n}, Q_{i, j}^{n}, N_{i, j}^{n}, C_{i, j}^{n}\right)\right) \\
C_{i, j}^{n+1} & =\frac{1}{1+\gamma_{1} P_{i, j}^{n}+\gamma_{2} Q_{i, j}^{n}}\left(1-\alpha\left(P_{i, j}^{n}+Q_{i, j}^{n}+N_{i, j}^{n}\right)\right)
\end{aligned}
$$

with $U_{i, j}^{n}$ and $V_{i, j}^{n}$ defined by

$$
\begin{aligned}
U_{i, j}^{n}= & \frac{\left(P_{i+1, j}^{n}-P_{i-1, j}^{n}\right) R_{i, j}^{n}\left(R_{i+1, j}^{n}-R_{i-1, j}^{n}\right)+4 P_{i, j}^{n} R_{i, j}^{n}\left(R_{i+1, j}^{n}-2 R_{i, j}^{n}+R_{i-1, j}^{n}\right)-P_{i, j}^{n}\left(R_{i+1, j}^{n}-R_{i-1, j}^{n}\right)^{2}}{2\left(h R_{i, j}^{n}\right)^{2}} \\
& +\frac{\left(P_{i, j+1}^{n}-P_{i, j-1}^{n}\right) R_{i, j}^{n}\left(R_{i, j+1}^{n}-R_{i, j-1}^{n}\right)+4 P_{i, j}^{n} R_{i, j}^{n}\left(R_{i, j+1}^{n}-2 R_{i, j}^{n}+R_{i, j-1}^{n}\right)-P_{i, j}^{n}\left(R_{i, j+1}^{n}-R_{i, j-1}^{n}\right)^{2}}{2\left(h R_{i, j}^{n}\right)^{2}} ;
\end{aligned}
$$




$$
\begin{aligned}
V_{i, j}^{n}= & \frac{\left(Q_{i+1, j}^{n}-Q_{i-1, j}^{n}\right) R_{i, j}^{n}\left(R_{i+1, j}^{n}-R_{i-1, j}^{n}\right)+4 Q_{i, j}^{n} R_{i, j}^{n}\left(R_{i+1, j}^{n}-2 R_{i, j}^{n}+R_{i-1, j}^{n}\right)-Q_{i, j}^{n}\left(R_{i+1, j}^{n}-R_{i-1, j}^{n}\right)^{2}}{2\left(h R_{i, j}^{n}\right)^{2}} \\
& +\frac{\left(Q_{i, j+1}^{n}-Q_{i, j-1}^{n}\right) R_{i, j}^{n}\left(R_{i, j+1}^{n}-R_{i, j-1}^{n}\right)+4 Q_{i, j}^{n} R_{i, j}^{n}\left(R_{i, j+1}^{n}-2 R_{i, j}^{n}+R_{i, j-1}^{n}\right)-Q_{i, j}^{n}\left(R_{i, j+1}^{n}-R_{i, j-1}^{n}\right)^{2}}{2\left(h R_{i, j}^{n}\right)^{2}} ; \\
R_{i, j}^{n}= & P_{i, j}^{n}+Q_{i, j}^{n} .
\end{aligned}
$$

Here $F(P, Q, N, C), G(P, Q, N, C)$, and $H(P, Q, N, C)$ are given by

$$
\begin{aligned}
F(P, Q, N, C)= & g(C) P(P+m)(1-P-Q-N) \\
& -f_{1}(C) P+f_{2}(C) Q-f_{3}(C) P, \\
G(P, Q, N, C)= & f_{1}(c) P-f_{2}(c) Q-h(c) Q, \\
H(P, Q, N, C)= & h(c) Q+f_{3}(c) P .
\end{aligned}
$$

The initial conditions are set as follows:

$$
\begin{aligned}
& p(x, y, 0)=e^{\left(-0.01(x-100)^{2}-0.01(y-100)^{2}\right)}, \\
& q(x, y, 0)=n(x, y, 0)=0, \\
& c(x, y, 0)=1 .
\end{aligned}
$$

4.1. Allee Effect. It is now well known that the growth curves for multicellular spheroids are characterized by doubling time which decreases with increasing growth until a stable saturation was attained ([7]). However, the original model proposed in ([32]) does not support this behavior. The solutions exhibit travelling wave solutions and the tumor continues to grow outward. In this section, we show that Allee effect plays an important role in the growth of avascular tumor. Essentially, as the density of proliferating cells drops, the weak Allee effect further slows down its growth, which overall slows down the growth of the tumor.

In Figure 6, we compare typical profiles of proliferating cells, quiescent cells, and necrotic cells at $t=$ 20. Starting with the same initial data, both models exhibit three layers of different cells, with a necrotic core inside. With Allee effect, the tumor size is significantly reduced.

Figure 7 shows individual profiles for proliferating cells, quiescent cells, and necrotic cells without Allee effect at time $t=20$.

In Figure 8, we show how the strength of Allee effect impacts the size of the tumor. Figure 8(a) shows the density of proliferating cells at $t=20$ without Allee effect. Figure 8(b) shows the density of proliferating cells at $t=20$ with weak Allee effect $(m=0.4)$. When the strength of Allee effect is stronger $(m=0.1)$, there is a significant reduction in tumor size as shown in Figure 8(c).
Figure 9 shows the impact of Allee effect on the profile of necrotic core.

In Figure 10, we study the impact of Allee effect on the size of the tumor at different time. We locate the peak of the proliferating cell density and use its position to estimate the radius of the tumor. The results are shown in Figure 10. We note that the radius of tumor grows linearly without Allee effect. It is significantly reduced when weak Allee effect is present. When the strength of Allee effect is stronger, however, the radius grows exponentially at the very beginning, then quickly becomes linearly, and eventually reaches a plateau.

4.2. Impact of Apoptotic Cell Death Rate. This section considers the effect of cell death from both the quiescent cells (necrosis) and proliferating cells (apoptosis). Comparing to small apoptosis rate $\left(k_{N}=0.01\right)$, we increase this rate to $k_{N}=0.1$. The profiles of different tumor cells at different times are shown in Figure 11. Higher level of apoptosis leads to fewer quiescent cells and more necrotic cells. The rim of proliferating cells still remains on the boundary. A closer look at the concentration of nutrient reveals that a higher level of apoptosis leads to higher nutrient concentration at the center of the spheroid. This is due to the fact that a higher level of apoptosis increases the amount of necrotic cells at the center, thus reducing the amount of viable cells.

4.3. Impact of Proliferation Rate. In this section, we simulate the system with a smaller proliferation rate $\beta=0.4$ and a larger proliferation rate $\beta=0.6$. A larger proliferation rate leads to a higher density in proliferating cells which will also lead to more quiescent cells and necrotic cells. The result is shown in Figure 12. For a better illustration, please see the heat map in Figure 13.

4.4. Impact of Oxygen Degradation Rate. To study the effects of oxygen on the growth of the avascular tumor, we compare tumor cell density profiles at different oxygen degradation rate at $k_{1}=0.03$ and $k_{1}=0.15$ at time $t=20$. The spreading speed of the tumor increases as the degradation rate gets higher. At $t=20$, the radius of the tumor is estimated to be 27 when $k_{1}=0.03$, comparing to 30 when $k_{1}=0.15$ (Figure 14). As the degradation rate of oxygen increases, we also notice a significant reduction on necrotic region. For a 


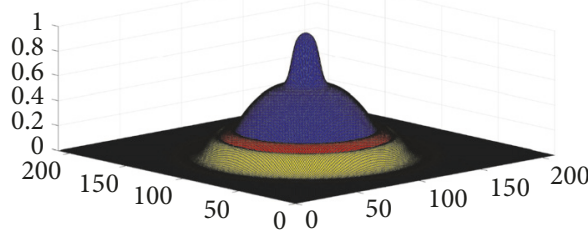

(a) Without Allee effect

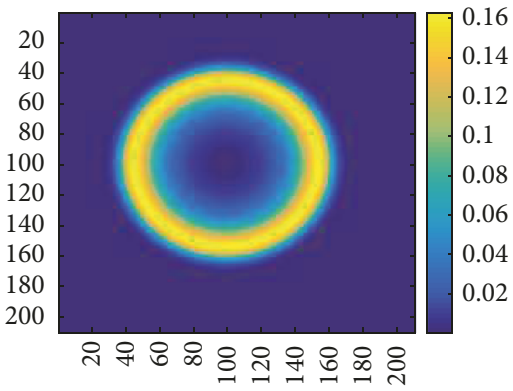

(c) Heat map of P without Allee effect

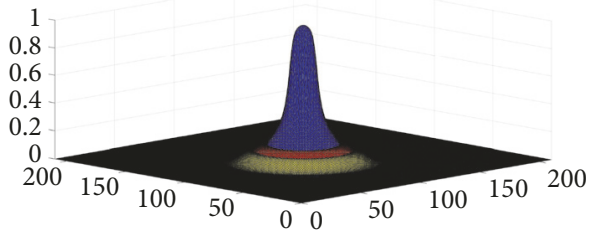

(b) With Allee effect

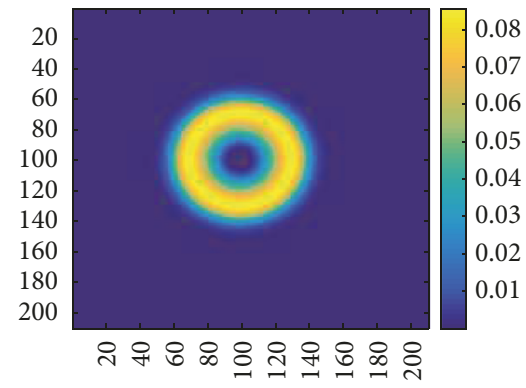

(d) Heat map of P with Allee effect

FIGURE 6: Impact of Allee effect: blue profile represents the density of the necrotic core; red profile represents the density of quiescent cells; the yellow profile represents the density of the proliferating rim.

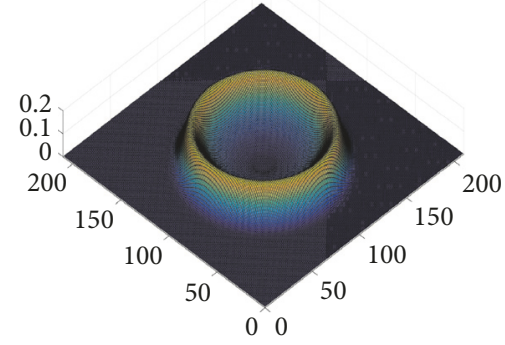

(a) Proliferating cells

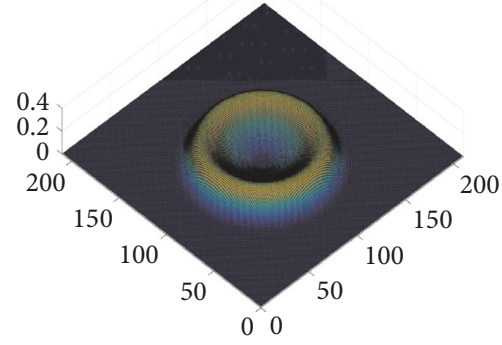

(b) Quiescent cells

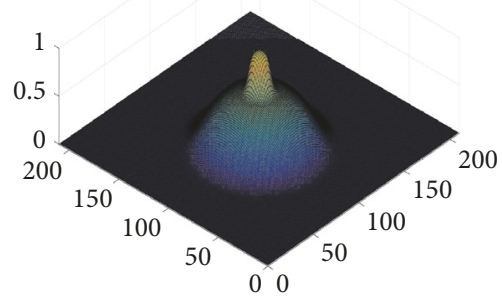

(c) Necrotic cells

FIGURE 7: Typical profile of proliferating cells, quiescent cells, and necrotic cells without Allee effect at $t=20$.

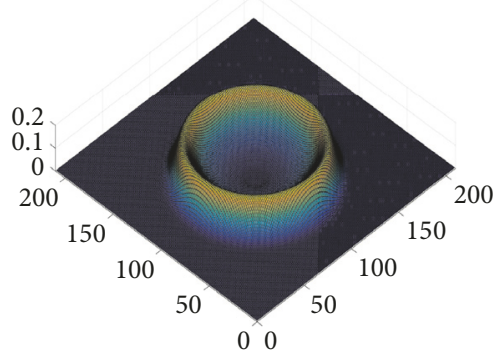

(a) No Allee effect

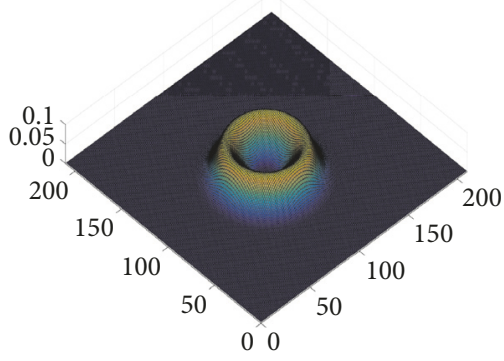

(b) Weak Allee effect

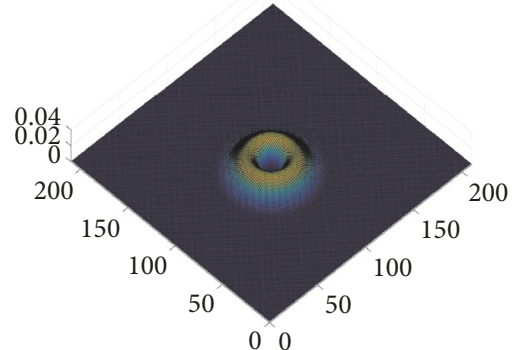

(c) Stronger Allee effect

FIGURE 8: Density of proliferating cells without Allee effect, with weak Allee effect $(m=0.4)$, and with stronger Allee effect $(m=0.1)$ at $t=20$.

better illustration, please see the heat map of all the cells in Figure 15.

4.5. Impact of Critical Oxygen Level. In this section we observe a very important relationship between the growth rate of the tumor and the critical oxygen level $C_{c r i t 1}$ for proliferation. To study the impact of critical oxygen level on the growth of the tumor, we calculate the tumor radius at $t=20$ for different critical values. The result is shown in Figure 16. The simulations show that the tumor growth 


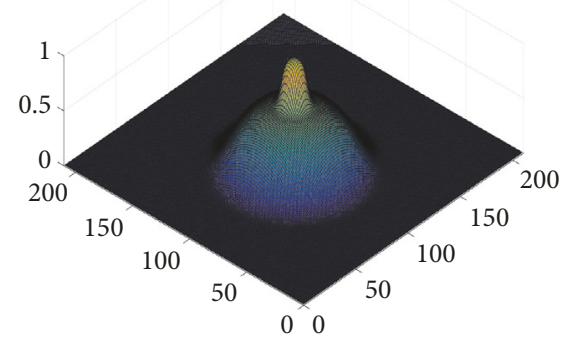

(a) No Allee effect

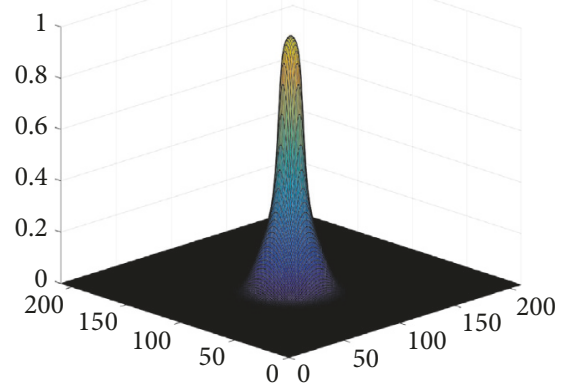

(b) Weak Allee effect

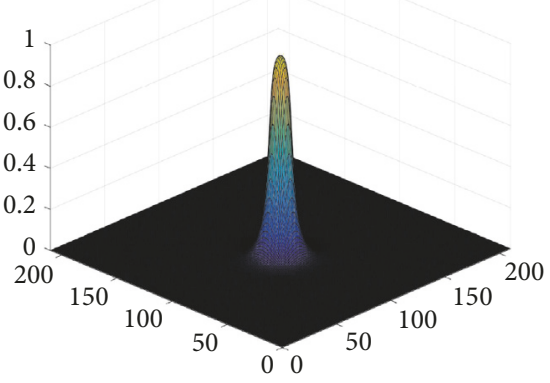

(c) Stronger Allee effect

FIGURE 9: Density of necrotic cells without Allee effect, with weak Allee effect $(m=0.4)$, and with stronger Allee effect $(m=0.1)$. $t=20$.

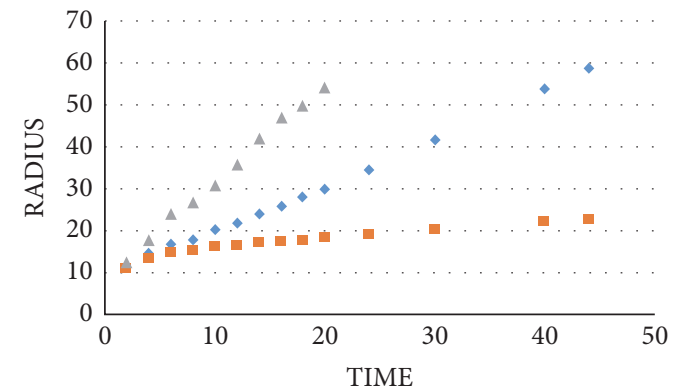

- Weaker Allee Effect

- Stronger Allee Effect

$\triangle$ No Allee Effect

FIGURE 10: The impact of weak Allee effect on the spreading speed of avascular tumor. If the weak Allee effect is strong, the growth of a tumor appears to be exponentially at the beginning, approximately linearly, and then reaches a plateau.

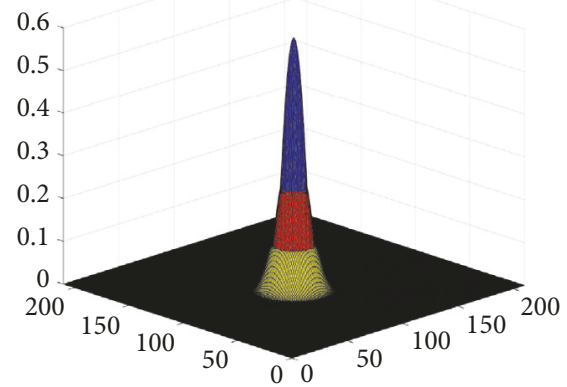

(a) Large apoptotic cell death at $t=3$

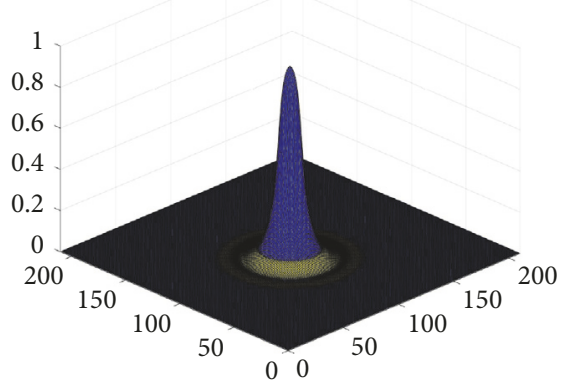

(b) Large apoptotic cell death at $t=10$

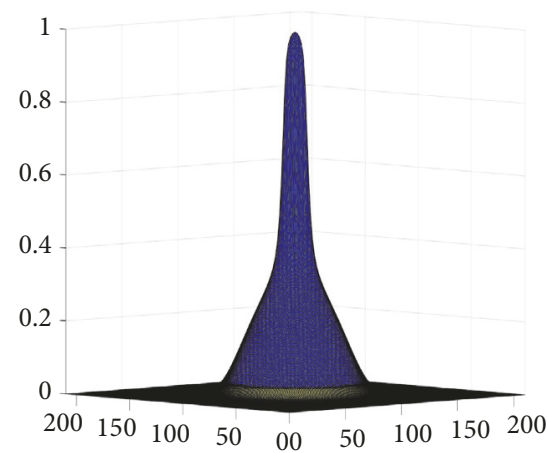

(d) Large apoptotic cell death at $t=30$

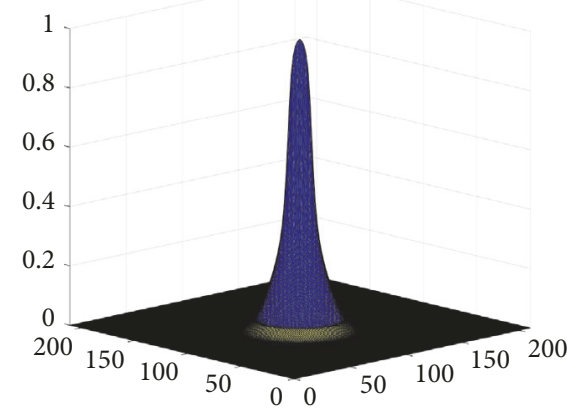

(c) Large apoptotic cell death at $t=20$

FIGURE 11: Large apoptotic cell death $\left(k_{N}=0.1\right)$ at different time. 


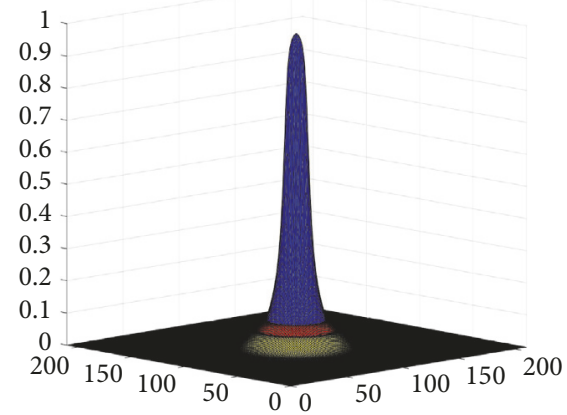

(a) Small proliferation rate

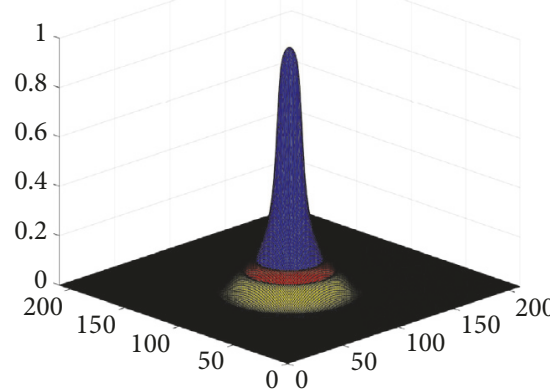

(b) Standard proliferation rate

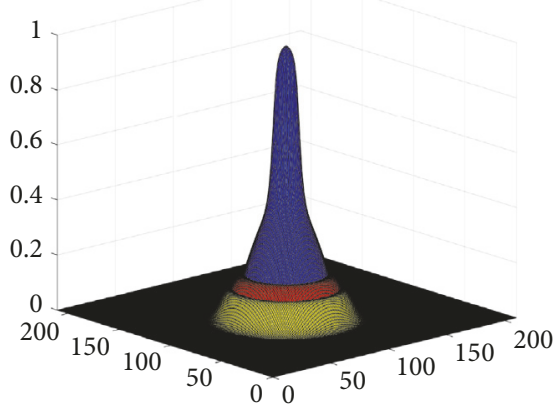

(c) Large proliferation rate

FIGURE 12: Impact of proliferation rate: (a) $\beta=0.4$, (b) $\beta=0.5$, and (c) $\beta=0.6$. For all three simulations, $t=20$.

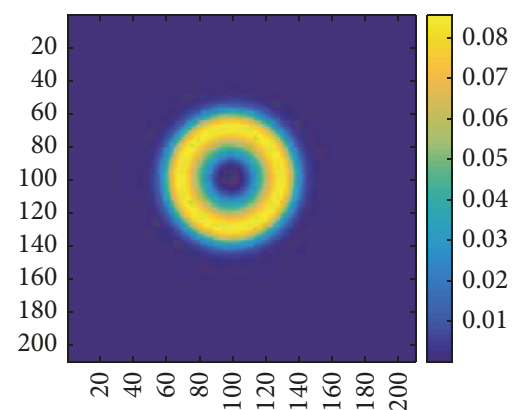

(a) Proliferating cells

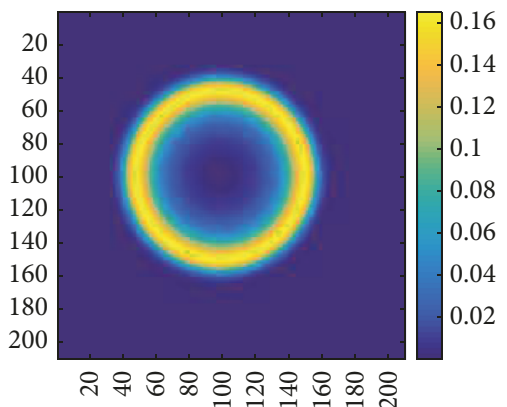

(d) Proliferating cells

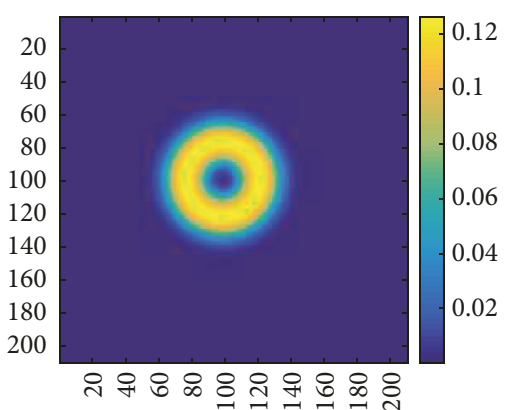

(b) Quiescent cells

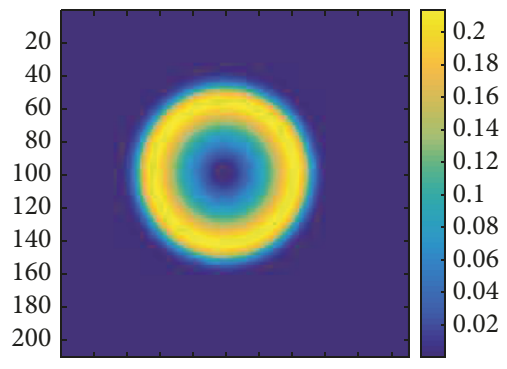

위요유요용

(e) Quiescent cells

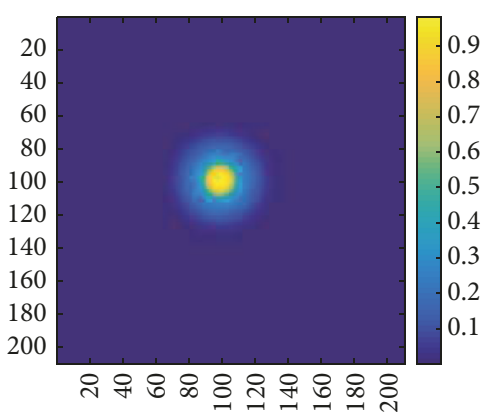

(c) Necrotic cells

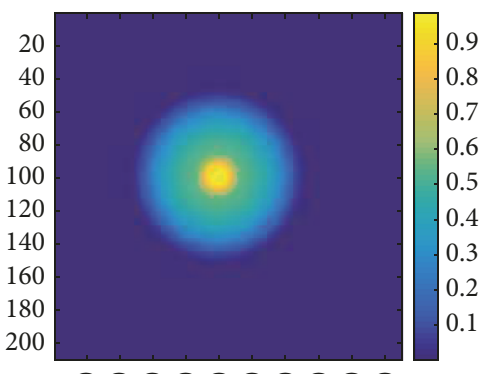

유우 \&\&으용유

(f) Necrotic cells

FIGURE 13: Heat map of cell densities at different proliferating rates: the top row corresponds to the standard rate at $\beta=0.4$; the bottom row corresponds to a higher proliferating rate at $\beta=0.6$.

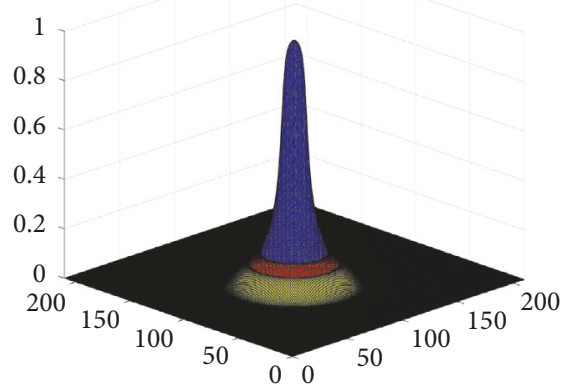

(a) Large oxygen degradation rate

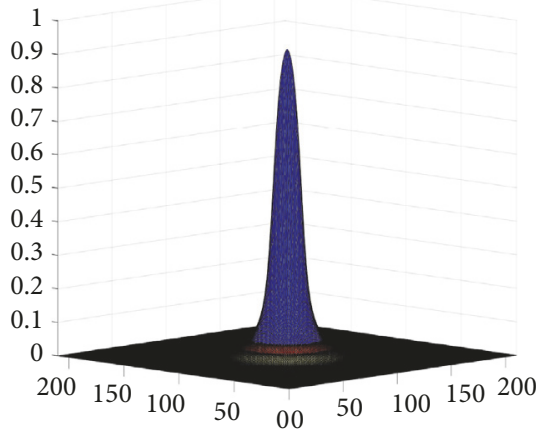

(b) Small oxygen degradation rate

FIGURE 14: Impact of oxygen degradation rate on the growth of the tumor at $t=20$ : (a) $k_{1}=0.15$; (b) $k_{1}=0.03$. 


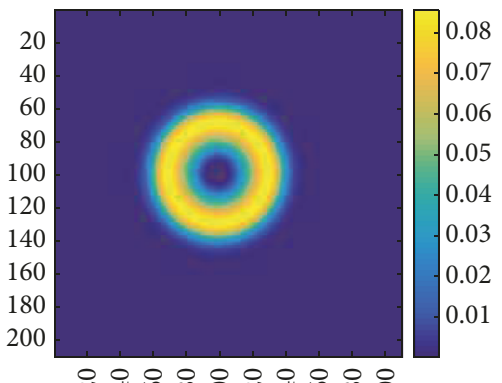

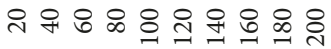

(a) Proliferating cells

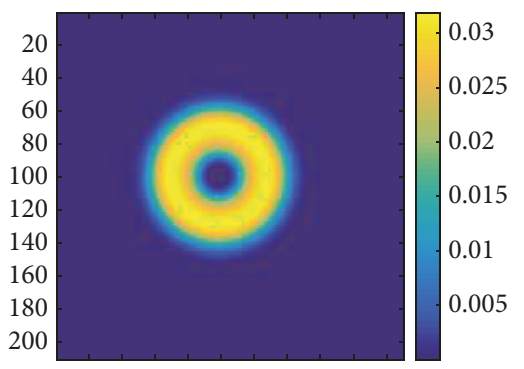

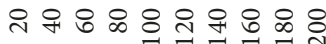

(d) Proliferating cells

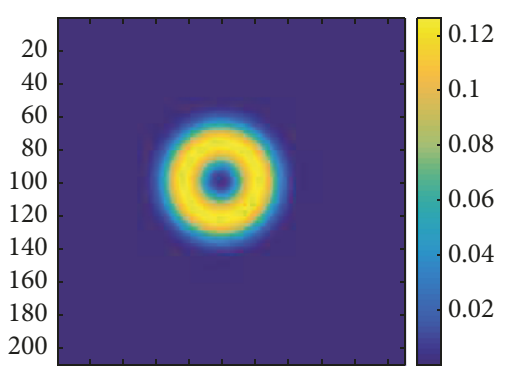

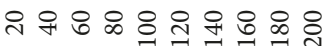

(b) Quiescent cells

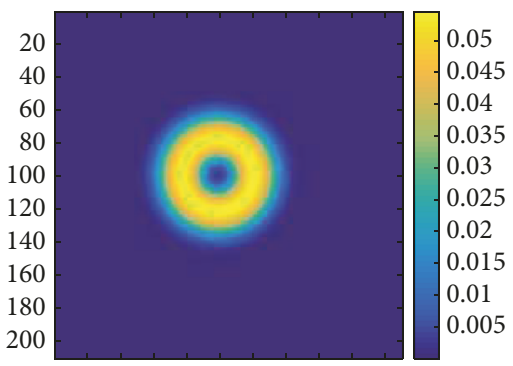

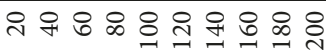

(e) Quiescent cells

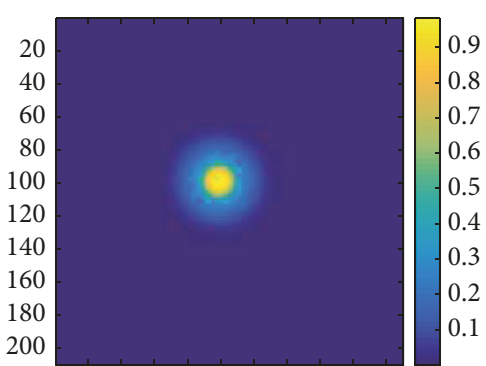

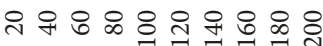

(c) Necrotic cells

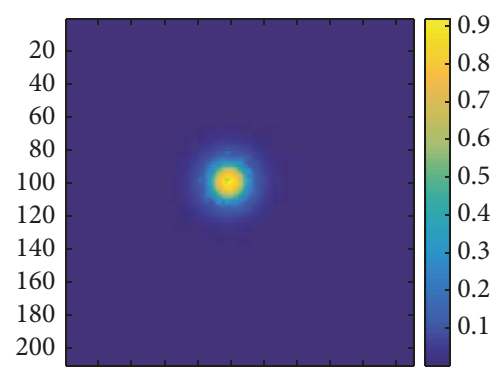

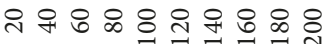

(f) Necrotic cells

Figure 15: Heat map of cell densities at different oxygen degradation rates: the top row corresponds to the standard rate at $k_{1}=0.15$; the bottom row corresponds to a lower degradation rate at $k_{1}=0.03$.

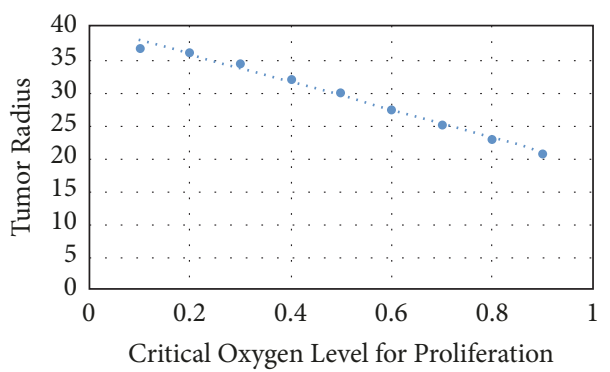

FIGURE 16: Dependence of tumor radius on critical oxygen concentration $c_{\text {crit } 1}$ for proliferation at time $t=20$ for the standard set of parameters.

speed decreases dramatically as the critical oxygen level increases.

4.6. Impact of Crowding Factor $\alpha$. This section compares the growth of the tumor layers for different crowding factors. A larger crowding factor $\alpha$, i.e., increasing the cell crowding, lowers the availability of oxygen. As a result, the tumor has a smaller density of proliferating cell and quiescent cell. The necrotic core of the tumor, however, becomes denser. Figure 17 shows the tumor profiles at $t=20$ for $\alpha=0.1$ and $\alpha=0.9$, respectively.

\section{Summary and Discussions}

This work is the first $2 \mathrm{D}$ mathematical model that incorporates Allee effect in the growth of avascular tumor. In the present paper we study the impact of various parameters on tumor progression. This work further expands our understanding of the process of avascular tumor growth by considering weak Allee effect and apoptosis as an additional mechanism of cell death. The extension to 2 spatial dimensions allows a more precise description of the processes of avascular tumor growth. One of our main findings is that weak Allee effect may lead to a plateau size. Another finding is that apoptosis increases the size of necrotic core and reduces the amount of viable cells. Additional numerical findings can be summarized as follows: (a) Large proliferation rate increases the overall size of tumor. (b) Large degradation rate of oxygen significantly reduces the size of necrotic region. (c) Increasing the critical oxygen concentration reduces the growth rate of the tumor. We point out that our aim in this work is not to propose completely new mathematical models, but to investigate two new mechanisms (Allee effect and 


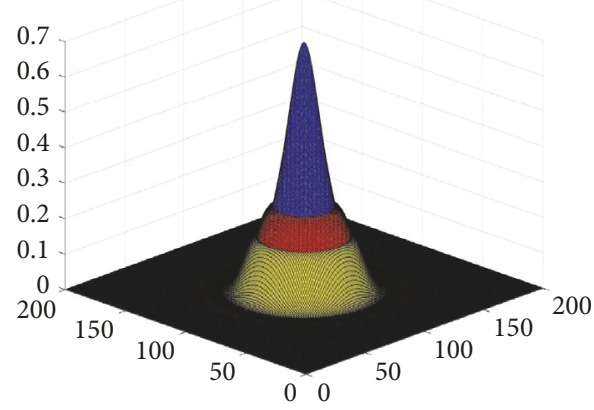

(a) Small crowding factor

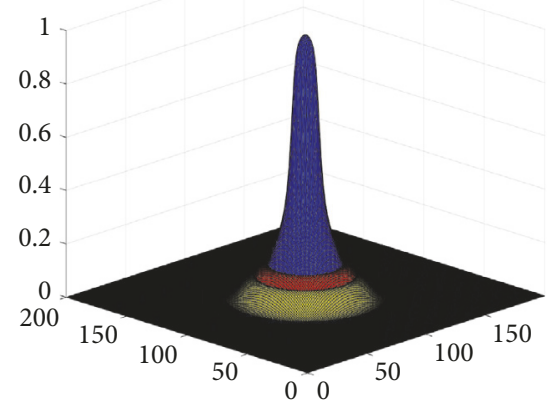

(b) Large crowding factor

FIGURE 17: Impact of crowding factor ( $\alpha=0.1$ vs $\alpha=0.9$ ) on the growth of tumor cells, $\mathrm{t}=20$.

apoptosis) through numerical simulations of Owen-Sherratt model. It is surely more realistic to consider the spheroid in 3D. But the main ingredient of the current research relies on the assumption that the nutrient reaches the core of the avascular tumor much faster than the reaction time of proliferating cells, etc. For $3 \mathrm{D}$ spheroid, this assumption no longer holds.

The impact of weak Allee effect on the growth of avascular tumors is investigated in this article. The understanding of this early stage is relevant to progression, relapse, and metastasis. A model that considers weak Allee effect may help to improve the predictions of tumor growth and relapse dynamics.

\section{Data Availability}

The data used to support the findings of this study are available from the corresponding author upon request.

\section{Disclosure}

The result in this article has been presented at 38th Southeastern-Atlantic Regional Conference on Differential Equations.

\section{Conflicts of Interest}

The authors have no conflicts of interest.

\section{References}

[1] R. Tiina, S. J. Chapman, and K. M. Philip, "Mathematical models of avascular tumor growth," SIAM Rev, vol. 69, pp. 179-208, 2007.

[2] L. J. Kleinsmith, Principles of Cancer Biology, Pearson Education, San Francisco, Calif, USA, 2005.

[3] T. Nederman, "Effects of vinblastine and 5-fluorouracil on human glioma and thyroid cancer cell monolayers and spheroids," Cancer Research, vol. 44, no. 1, pp. 254-258, 1984.
[4] D. I. Wallace, A. Dunham, P. X. Chen et al., "A model for spheroid versus monolayer response of SK-N-SH neuroblastoma cells to treatment with 15-Deoxy-PGJ2," Computational and Mathematical Methods in Medicine, vol. 2016, 2016.

[5] M. Zanoni, F. Piccinini, C. Arienti et al., “3D tumor spheroid models for in vitro therapeutic screening: a systematic approach to enhance the biological relevance of data obtained," Scientific Reports, vol. 6, Article ID 19103, 2016.

[6] J. Carlsson, K. Nilsson, B. Westermark et al., "Formation and growth of multicellular spheroids of human origin," International Journal of Cancer, vol. 31, no. 5, pp. 523-533, 1983.

[7] J. P. Freyer, "Role of necrosis in regulating the growth saturation of multicellular spheroids," Cancer Research, vol. 48, no. 9, pp. 2432-2439, 1988.

[8] P. Szlosarek, K. A. Charles, and F. R. Balkwill, “Tumour necrosis factor- $\alpha$ as a tumour promoter," European Journal of Cancer, vol. 42, no. 6, pp. 745-750, 2006.

[9] L. A. Kunz-Schugart, M. Kreutz, and R. Knuechel, "Multicelluar spheriods: a three-dimensional in vitro culture system to study tumor biology," International Journal of Experimental Pathology, vol. 79, no. 1, 1998.

[10] J. Folkman and M. Hochberg, "Self-regulation of growth in three dimenions," Journal of Experimental Medicine, vol. 138, no. 4, pp. 745-753, 1973.

[11] D. I. Wallace and X. Guo, "Properties of tumor spheroid growth exhibited by simple mathematical models," Frontiers in Oncology, vol. 3, article no. 51, 2013.

[12] W. F. MuellerKlieser, "Tumor biology and experimental therapeutics," Critical Review in Oncology/Hematology, vol. 36, no. 2-3, pp. 123-139, 2000.

[13] W. Mueller-Klieser, J. P. Freyer, and R. M. Sutherland, "Influence of glucose and oxygen supply conditions on the oxygenation of multicellular spheroids," British Journal of Cancer, vol. 53, no. 3, pp. 345-353, 1986.

[14] J. T. Rutka, J. R. Giblin, M. E. Berens et al., "The effects of human recombinant tumor necrosis factor on glioma-derived cell lines: cellular proliferation, cytotoxicity, morphological and radioreceptor studies," International Journal of Cancer, vol. 41, no. 4, pp. 573-582, 1988.

[15] I. Kareva, "What can ecology teach us about cancer?" Translational Oncology, vol. 4, no. 5, pp. 266-270, 2011. 
[16] K. S. Korolev, J. B. Xavier, and J. Gore, "Turning ecology and evolution against cancer," Nature Reviews Cancer, vol. 14, no. 5, pp. 371-380, 2014.

[17] D. Basanta and A. R. A. Anderson, "Exploiting ecological principles to better understand cancer progression and treatment," Interface Focus, vol. 3, no. 4, 2013.

[18] W. C. Allee and E. S. Bowen, "Studies in animal aggregations: mass protection against colloidal silver among goldfishes," Journal of Experimental Zoology, vol. 61, no. 2, pp. 185-207, 1932.

[19] P. C. Huszthy, I. Daphu, S. P. Niclou et al., "In vivo models of primary brain tumors: Pitfalls and perspectives," NeuroOncology, vol. 14, no. 8, pp. 979-993, 2012.

[20] D. Tilkorn, A. Daigeler, I. Stricker et al., "Establishing efficient xenograft models with intrinsic vascularisation for growing primary human low-grade sarcomas," Anticancer Reseach, vol. 31, no. 12, pp. 4061-4066, 2011.

[21] K. Böttger, H. Hatzikirou, A. Voss-Böhme et al., "An emerging Allee effect is critical for tumor initiation and persistence," PLoS Computational Biology, vol. 11, no. 9, Article ID e1004366, 2015.

[22] Z. Neufeld, W. von Witt, D. Lakatos, J. Wang, B. Hegedus, and A. Czirok, "The role of Allee effect in modelling post resection recurrence of glioblastoma," PLoS Computational Biology, vol. 13, no. 11, 2017.

[23] K. A. Rejniak and A. R. A. Anderson, "A computational study of the development of epithelial acini: I. Sufficient conditions for the formation of a hollow structure," Bulletin of Mathematical Biology, vol. 70, no. 3, pp. 677-712, 2008.

[24] H. B. Frieboes, F. Jin, Y.-L. Chuang, S. M. Wise, J. S. Lowengrub, and V. Cristini, "Three-dimensional multispecies nonlinear tumor growth-II: Tumor invasion and angiogenesis," Journal of Theoretical Biology, vol. 264, no. 4, pp. 1254-1278, 2010.

[25] P. Gerlee and A. R. A. Anderson, "Diffusion-limited tumour growth: simulations and analysis," Mathematical Biosciences and Engineering, vol. 7, no. 2, pp. 385-400, 2010.

[26] X. Li, V. Cristini, Q. Nie, and J. S. Lowengrub, "Nonlinear threedimensional simulation of solid tumor growth," Discrete and Continuous Dynamical Systems - Series B, vol. 7, no. 3, pp. 581604, 2007.

[27] R. P Araujo and D. L. S. McElwain, "A history of the study of solid tumor growth: the contribution of mathematical modelling," Bull. Math. Biol, vol. 66, no. 5, p. 1039, 2004.

[28] T. Roose, S. J. Chapman, and P. K. Maini, "Mathematical models of avascular tumor growth," SIAM Review, vol. 49, no. 2, pp. 179208, 2007.

[29] L. Sewalt, K. Harley, P. van Heijster, and S. Balasuriya, "Influences of Allee effects in the spreading of malignant tumours," Journal of Theoretical Biology, vol. 394, pp. 77-92, 2016.

[30] J. Ward and J. R. King, "Mathematical modelling of avasculartumour growth," Mathematical Medicine and Biology: A Journal of the IMA, vol. 14, no. 1, pp. 39-69, 1997.

[31] J. Ward and J. R. King, "Mathematical modelling of avasculartumour growth II: modelling growth saturation," Mathematical Medicine and Biology: A Journal of the IMA, vol. 16, no. 2, pp. 171-211, 1999.

[32] J. A. Sherratt and M. A. J. Chaplain, "A new mathematical model for avascular tumour growth," Journal of Mathematical Biology, vol. 43, no. 4, pp. 291-312, 2001.

[33] H. Y. Zhu and C. H. Ou, "Existence of traveling wavefronts for Sherratts avascular tumor model," Chaos, Solitons \& Fractals, vol. 44, pp. 218-225, 2011.
[34] M. Bertsch, D. Hilhorst, H. Izuhara, and M. Mimura, "Travelling wave solutions of a parabolic-hyperbolic system for contact inhibition of cell-growh," European Journal of Applied Mathematics, vol. 26, no. 3, pp. 297-323, 2015.

[35] A. Okubo and S. A. Levin, Diffusion and Ecological ProblemsModern Perspectives, Springer, 2nd edition, 2001.

[36] D. G. Aronson and H. F. Weinberger, "Multidimensional nonlinear diffusion arising in population genetics," Advances in Mathematics, vol. 30, no. 1, pp. 33-76, 1978.

[37] P. C. Fife, Lecture Notes in Biomathematics: Mathematical Aspects of Reacting and Diffusing Systems, vol. 28, SpringerVerlag, 1979.

[38] P. M. Altrock, L. L. Liu, and F. Michor, "The mathematics of cancer: integrating quantitative models," Nature Reviews Cancer, vol. 15, no. 12, pp. 730-745, 2015.

[39] J. P. Freyer, E. Tustanoff, A. J. Franko, and R. M. Sutherland, "In situ oxygen consumption rates of cells in V-79 multicellular spheroids during growth," Journal of Cellular Physiology, vol. 118, no. 1, pp. 53-61, 1984.

[40] M. A. J. Chaplain and G. Lolas, "Mathematical modelling of cancer cell invasion of tissue: The role of the urokinase plasminogen activation system," Mathematical Models and Methods in Applied Sciences, vol. 15, no. 11, pp. 1685-1734, 2005.

[41] M. A. J. Chaplain and G. Lolas, "Standard mathematical modelling of cancer invasion of tissue: Dynamic heterogeneity," Networks and Heterogeneous Media, vol. 1, no. 3, pp. 399-439, 2006.

[42] S. Petrovskii, A. Morozov, and B.-L. Li, "Regimes of biological invasion in a predator-prey system with the Allee effect," Bulletin of Mathematical Biology, vol. 67, no. 3, pp. 637-661, 2005. 


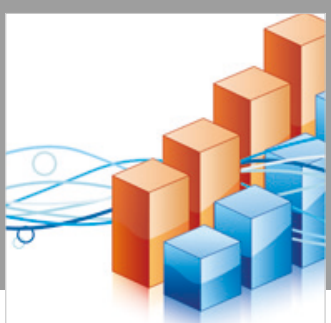

Advances in

Operations Research

\section{-n-m}
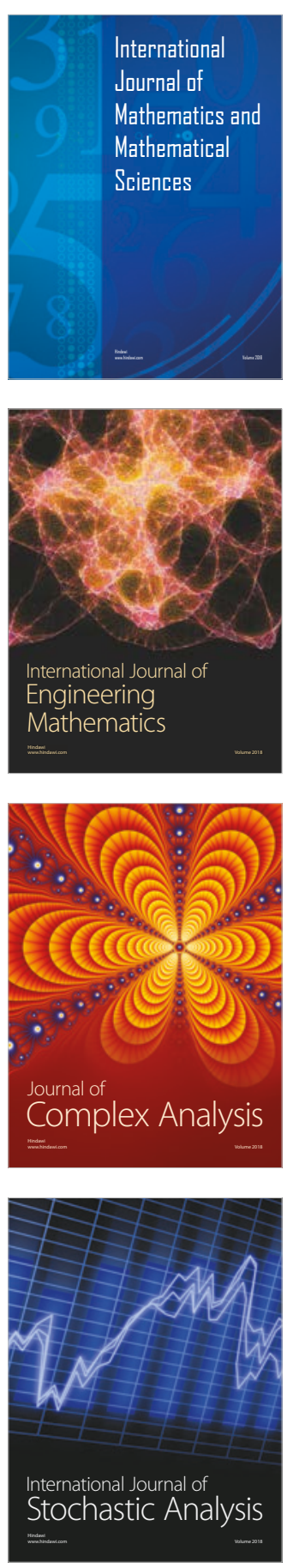
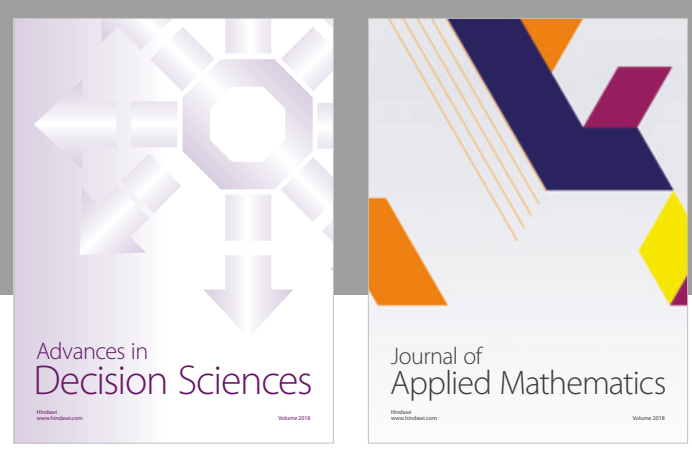

Journal of

Applied Mathematics
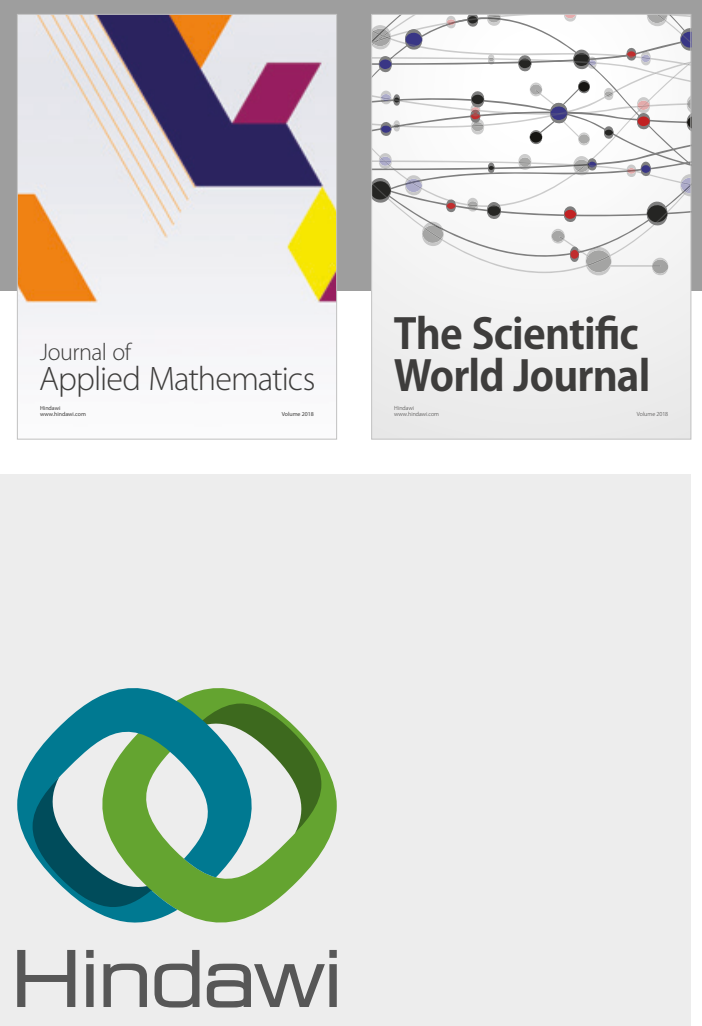

Submit your manuscripts at

www.hindawi.com

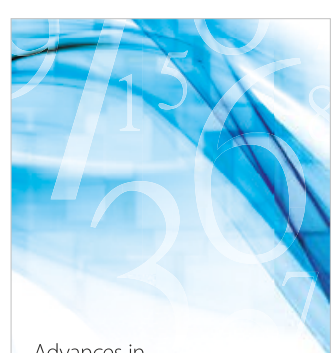

Advances in
Numerical Analysis
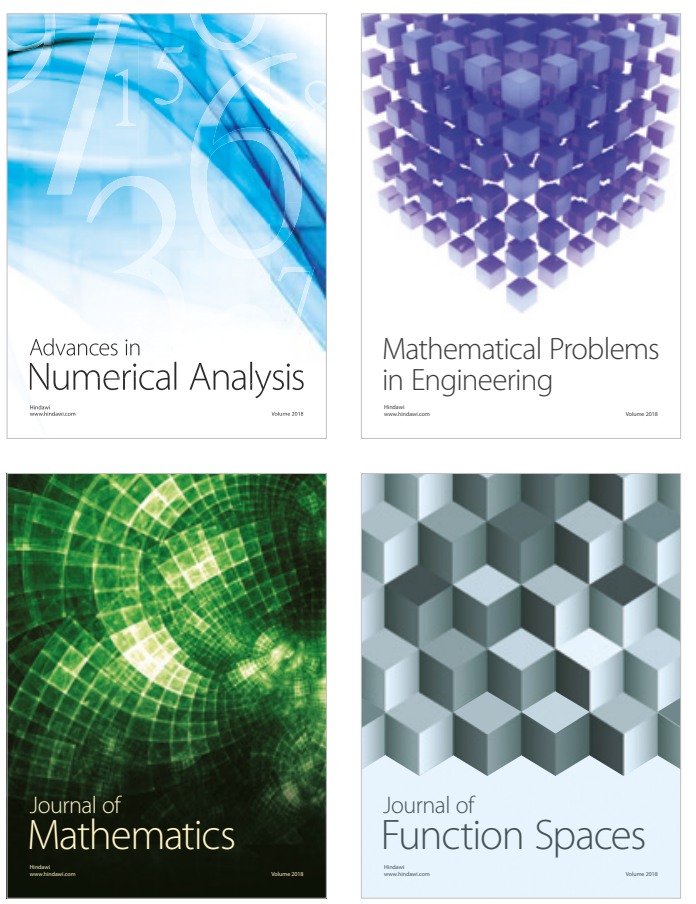

Mathematical Problems in Engineering

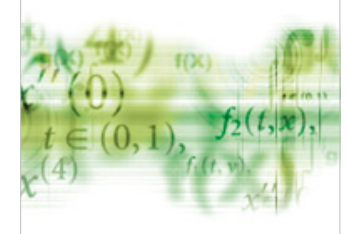

International Journal of

Differential Equations

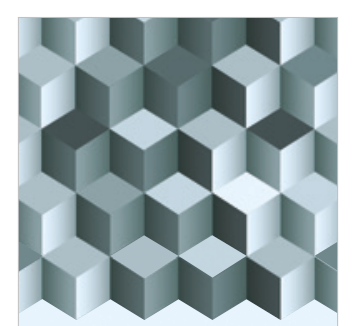

Journal of

Function Spaces
The Scientific

World Journal

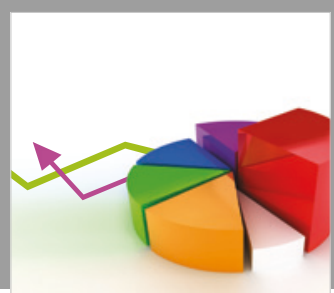

Journal of

Probability and Statistics
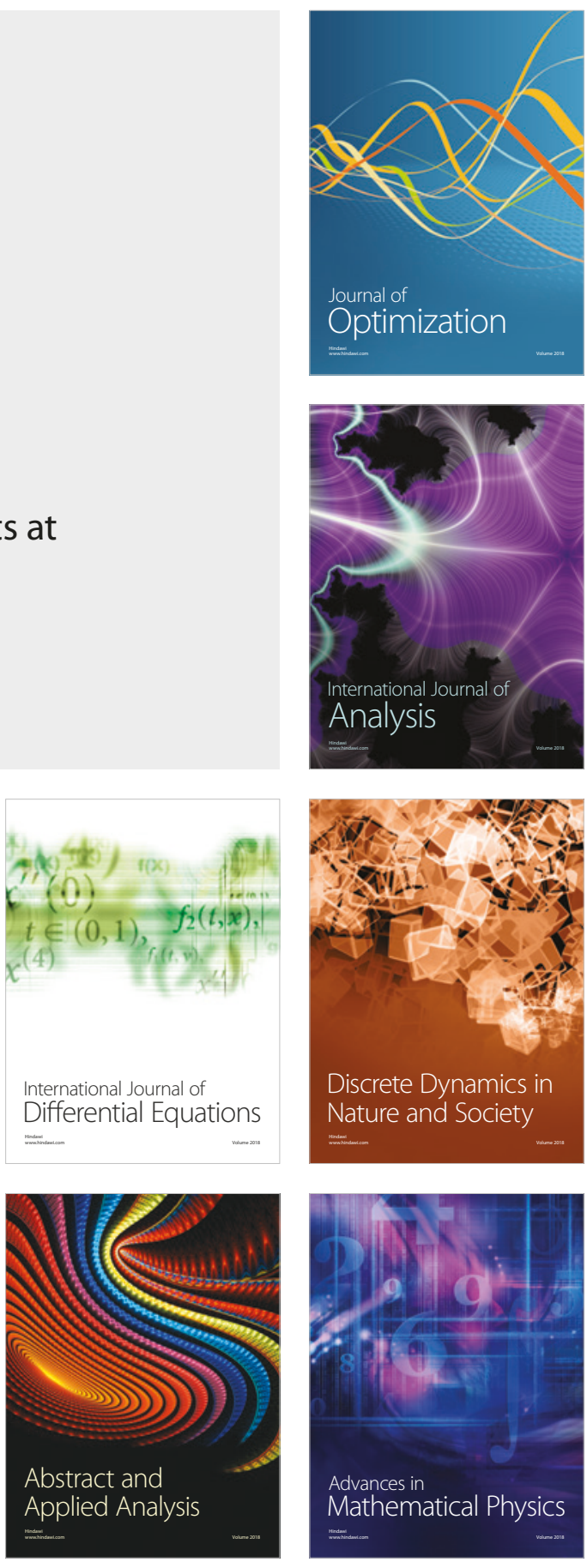